Letras, Lima, Univ. San Marcos. 63 (91): 97-105, 1992

\title{
Hemingway: el viejo y un mar de palabras
}

MIGUEL ANGEL. HUAMAN

La crítica norteamericana ha desarrollado toda una concepción general de aproximación a la obra de Ernest Hemingway sesgada por la extraordinaria imagen pública que todavía goza el inefable "Papa". Subsidiaria del peso comercial de una fama individual acrecentada con la muerte del autor, Philip Young y Carlos Baker, dos de los más representativos críticos hemingwanianos, privilegian un enfoque basado en "la confusión entre el autor-creador, que pertenece $a$ lo obra, $y$ el cutor-real, que es un elemento en el acontecer ético y seciat de qa vida" (1).

Intentaremos establecep lestion adtitud nerítica " absolutamente infundada y que conduce a un predominio de lo fáctico o evidente en la valoración de la obra de Hemingway a partir de una reflexión general sobre sus novelas, prestando una atención especial a El viejo y el mar (2), y centrando nuestro análisis en el personaje y su cconlecer estético.

Dentro de la producción narrativa de E.H., la crítica norteamericana casi unánimemente ha considerado a Fiesta (The sun also rises, 1926) y Adiós a las armas (A farewell to arms, 1929) conjuntamente con El viejo y el mar (The old man and the sea) como las novelas más significativas e importantes, es decir como

(1) BAJTIN, M.M.: Estética de la creación verbal. Siglo XXI, México, 1982, p. 18.

(2) Hemos manejado la versión española autorizada por el autor, editada por Populibros Peruanos, que es la misma aparecida en Ediciones Kraft (Bs. As. 1954) y recientemente reeditada por Editorial Oveja Negra (Colombia, 1984). 
sus obras mayores. Aunque en el balance final es perceptible cierta priorización de las dos primeras en detrimento de esta última (3).

Creemos, sin embargo, que El viejo y el mar (EVM) ha sido poco comprendida en su real significación, pese a las numerosas alusiones y menciones sobre ella, precisamente por esta actitud a la que son proclives los principales críticos de E.H., de enfatizar lo biográfico y personal en las obras anteriores.

EVM es la obra menos autobiográlica de Hemingway en opinión de estos entendidos $y_{1}$ sin duda, pese al mérito que se le reconoce, no se ha profundizado en las virtudes estéticas de la misma y sobre el peso de la obra en el conjunto de la labor de su cutor.

Retomaremos con carácter explicalivo algunas opiniones de la crítica norteamericana sobre la obra de E.H. en general y sobre EVM en particular a fin de analizar luego, con los conceptos de M.M. Bajtín, la narrativa de Hemingway con un disianciamiento conceptual frente a las distorsiones que creemos rigen los enfoques todavía vigentes en torno a E.H.

\section{E.H.: Dos personajes, tres motivos y algunos símbolos}

Philip Young habla de Nick Adams, personaje que aparece en "Campamento indio" (Indian Camp) el primer relato de $\mathrm{He}$ mingway de su libro de cuentos En nuestro tiempo (In our time, 1925), como el protagonista de muchas de sus obras "tan parecidos entre sí que hemos llegado a hablar de ellos en singular" (4). Es el héroe heridopque defino uno de los dos personajes claves de la obra conjunta de E.H., que aparecerá constantemente y que, como veremos, vendria a lserine proyedción literaria del propio Hemingway. El otro personaje recurrente sería el héroe norma, que por autonomía y ejemplo posibilita saturar, curar, las heridas o deficiencias del anterior y que como figura "no es el propio Hemingway disfrazado" (5).

Además del héroe de Hemingway y del héroe norma, Young nos presenta tres motivos centrales: la herida, la ruptura con la sociedad y la norma, cuyos ajustes recíprocos "son el cisunto de toda la obra significativa de Hemingway" (6).

(3) Por ejemplo, Philip Young dice en el libro: Tres escritores norteamericanos, Madrid, Gredos, 1961, p. 16: "The sun also rises sigue siendo una de las mejores novelas que haya escrito. La otra es su s1guiente libro A farewell to arms".

(4) YOUNG, P., ob. cit., p. 8.

(5) YOUNG, P., ob. cit., p. 12.

(6) Ibid., p. 18. 
En el análisis de la obra narrativa de E.H. se van a mezclar definiciones y variaciones en torno a estos motivos y personajes, dilucidados a partir de las referencias a la vida del autor. Así JakeBernes, el héroe de Fiesta, tendrá una herida literal y simbólica, que en Frederic Henry protagonista de Adiós a las armas lleva a referir casi exactamente al propio E.H. que como estos héroes fue herido en la guerra al prestar servicio en una ambulancia.

Otra coincidencia que se establece entre la vida de E.H. y sus personajes está centrada en la protagonista que acompaña al héroe herido hemingwaniano: Bret Ashley y Katherine Barkley serían reencarnaciones literarias de Duff Twynaden, participante de un grupo semejante al que en Fiesta viaja a Pamplona, y Ägnes Hannah von Kurowsky, enfermera de la que se habría enamo. rado el joven Hemingway durante su convalecencia en el hospital.

Por otro lado, la crítica norteamericana, ha encontrado diversos símbolos cuyo sustento se establece a partir del conocimiento del Hemingway real. "La trampa biológica" y "La paz por separado" sólo se entienden, por ejemplo, como actitudes enfrentadas al destino que se ensaña con aquellos que sabiendo perdida la batalla de la existencia, por la inevitabilidad de la muerte, se muestran dignos en la desgracia ("grace under pressure"), opción existencial sin duda impregnada de la idea afirmada por $\mathrm{He}$ mingway en el sentido de que "la mejor preparación para un buen escritor reside en una infancia desgraciada" (7).

Se discuten así las obras de E.H. en función del autor-real queriendo ver ya una actitud antisemila (Robert Cohn), una alusión cristiana (Santiago) Bo un iemor a la oscuridad (Jake Barnes), propias de Hemingway y plasmadas en las obras. Asimismo, se asume una actitud "pasiar Prentedeli hedheliferario de las obras, punto que intentaremos precisar brevemente.

\section{E.H.: ¿Pesca mayor literaria?}

La crítica norteamericana habla de las virtudes literarias de la obra de Hemingway en términos de "estilo", como diría Buffón en este caso también "el estilo es el hombre", así Philip Young dice que "lo que más se ha imitado en Hemingway es el estilo de su prosa" y establece como principal característica "una delicada sencillez en el vocabulario y en la estructura de la frase. Las palabras usadas son normalmente cortas y corrientes, de lo que resulia una austera sobriedad y una curiosa frescura" (8).

Por otro lado se le reconoce como un maestro del diálogo pues "Hemingway tiene un oído que coge, como una trampa, todos los

(7) Ibid., p. 26.

(8) Ibid., p. 40. 
acentos y expresiones del humano lenguaje" (9), dando vida rá pidamente al personaje al reducir el diálogo al lenguaje espontáneo; un patrón esencial de expresiones y respuestas características del habla nos produce una ilusión de realidad superior a la que la propia realidad nos daría.

Todo lo señalado otorga a su narrativa un marcado aliento práctico y actuante, nada cerebral y descarnado, sus personajes por ello generalmente se definen por su acción y los sucesos, poco frecuente son las explicitaciones de intenciones en monólogos largos o cargados de atmósferas subjetivas. La visión es casi ab. jetiva y cinemctográfica, pudiendo apreciarse una suerte de incremento de la descripción o la subjetividad entre sus novelas cronológicamente, pero sin percler el sentido general que rige a toda su prosa.

Por otro lado, las estructuras de las novelas suelen esiablecerse en base a coniradicciones, alternancia de vida y muerte en Adiós alas armas, España y París en Fiesta, triunfo y derrota en El viejo y el mar. También, los finales generalmente se establecen bajo el predominio de la continuidad, sin intentar efectos contundentes.

Pese a todo lo señalado, existe una suerte de ambigüedad que recorre toda la obra narrativa de Hemingway, presente en las dudas o diversidad interpretativa de sus obras: novela pacifista - bélica, tema de amor o de guerra, generación perdida o tragedia del terruño (10). Fraceionamiento que puede referirse a una incitación a participar en la lectura o simplemente evidencia de la pérdida del controß del lenguaje.

Este estilo de Hemingway que es una incitación a la participación del lector" "afraves de los vacios voluntarios que su prosa contiene" (11) nos permite establecer un enfoque especial para el abordaje de la obra narrativa de E. H.

\section{E.H.: Autor y héroe}

Todo proceso literario trata de plasmar, en el acto de su producción creativa un aspecto individual fáclico entendido como la permanente caducidad del ser $\mathrm{y}$ un aspecto general ideal entendido como la adquisición efímera de la universalidad del hacer. En la confrontación de estos aspectos a partir del texto literario y su materialidad real lingüística percibimos el valor de la escritura como efecto estético.

\section{(9) Ibid., p. 40}

(10) cf. WILLIAMS WIRT: El arte trágico de Ernest Hemingway, Edamex, México, 1983.

(11) CASTELLET, Josep Ma. Prólogo al libro Hemingway de Antony Burgers, Salvat Editores S.A., Barcelona, 1986, p. 14. 
Este proceso literario obtiene su concreción en la producción creativa de un individuo: el autor. Por más que sea imposible desligar al individuo de un entorno social que le sirve de matriz nutricia, consciente o inconscientemente, el proceso literario marca la siempre aludida soledad del escritor en la medida que su performance o desempeño, su trabajo, responde a una motivación que sólo puede darse, por lo menos en esta realidad social que compartimos y nos condiciona, en términos personales. De chí el hecho de que la producción literaria como categoría social posea unce objetiva realización estrictamente individual y que sea el individuo, el escritor, el único que ponga en ejercicio, en funcionamiento de una manera esencialmente aislada un hecho social: la escritura.

El autor se enfrenta al hecho creativo con una actitud arquitectónicamente estable y dinámicamente viva, dualidad plasmada en el personaje y comprende una toialidad en base a la reaqción frente a las manifestaciones aisladas de la caracterización. "La lucha de un artista por una imagen definida y esiable de un personaje es, mucho, una lucha consigo mismo" (12).

Sin embargo, este enfrentamiento como proceso no podemos abordarlo de una manera inmediala pues sólo tenemos la objetiva plasmación del personaje en la obra. Interrogarnos sobre las molivaciones psicológicas y por el desenvolvimiento causal de dicho proceso no pertenece al ámbito de nuestro interés. Opiniones y datos biográficos acerca de este proceso de plasmación en la creación son materiales que pueden adquirir valor crítico sólo "después que sea ilegible el sentido artístico de la obra" (13).

Existe un principlo decoereleclof enfre elpersonaje y el cutor que no debe buscarse lege lenexplicatcion deeles obra mediante datos biográficos (o sea las simples coincidencias en los hechos de la vida del personaje con los del autor) pues sólo esián referidos a la unidad entre vida psicológica y social, que no brinda una comprensión estético formal de la totalidad arlística de la obra que se encuentra en un nivel diferente.

Definamos, por lo tanto, autor y personaje como correlatos de la totalidad artística de una obra:

Autor: es "quien confiere la unidad activa e intensa a la totalidad concluida del personaje y de la obra; esta unidad se extrapone a cada momento determinado de la obra" (14).

Personaje: Es quien vive cognoscitiva y éticamente dentro de un mundo determinado por la conciencia. "La conciencia del personaje, su modo de sentir y desear al mundo (su orientación emocio-

(12) BAJTIN, M. M. Ob. cit., p. 14

(13) Ibid., p. 15.

(14) Ibid., p.20. 
nal y volitiva) están encerrados como por un anillo por la conciencia abarcadora que posee el autor con respecto a su personaje y su mundo" (15).

Queda establecido que la conciencia del autor es conciencia de la conciencia, pues abarca al personaje y a su propio mundo de conciencia. Por ello la unidad del personaje, como totalidad conclusiva (formal y semánticamente) no puede aparecer desde el interior del personcje sino que desciende de otra conciencia (creadora) por medio de momentos que se extraponen a la conciencia misma, es decir que transgreden la conciencia real del personaje y su conciencia posible.

"El interés vital (ético y cognoscitivo) del personaje eslá comprendido por el interés artístico del autor. En este sentido, la objetividad estética se mueve en un sentido diferente con respecto a la objetividad cognoscitiva y ética esta última es una apreciación imparcial y desapasionada de una persona y de un suceso determinados desde el punto de vista común o tenido por tal, que aspira a ser un valor universal, ético y cognoscitivo; para la objetividad estética, el centro valorativo es la totalidad del personaje y del suceso que le concierne, a los cuales se les subordina todos los valores éticos y cognoscitivos: la objetividad ética y estética abarca e incluye a la objetividad ético-cognoscitiva" (16).

El autor debe encontrar un punto de apoyo fuera de sí mismo para que la unidad llegue a ser un fenómeno estético concluso, si el autor pierde este punto valorativo de la extraposición ello es captado por el lector y se matericliza en un efecto estético disonante, característicg presente en la obfa de Hemingway como veremos. Asimismo es Inedesatio-establecer los Casos típicos de desviación de la actityd direqta deblquton cons respecio a su personaje, que tiene lugar cuando el personaje coincide con el autor, es decir cuando existe una fuerte carga autobiográfica como es el caso del propio Hemingway.

E.H.: Tres novelas, tres actitudes.

El primer caso que establece Bajtín es aquél donde "el personaje se apropia del autor. La orientación emocional y volitiva del personaje, su postura ética y cognoscitiva posee tanto prestigio para el autor que éste no puede dejar de ver el mundo de abjetos sin usar la visión de su personaje".

Creemos que éste es el caso de la primera de las novelas indicadas anteriormente: Fiesta. ¿Cuál es el personaje principal? Jake Barnes, Bret Ashley o la post-guerra; cualquiera de ellos ejer

(15) Ibid., p. 22.

(16) Ibid., p. 20. 
ce indudablemente frente al autor una suerle de hegemonía, de primado. Es innegable la actitud confusa y contradictoria del propio Hemingway frente a la guerra, por ejemplo (criticar, participar); también su visión frente a la mujer y su machismo (dominante-dominado); y, por último, la afectación personal del trabajo periodístico. Ásí, dicho prestigio, es posible trasladarlo a la forma como encara dichos protagonismos, aunque es posible encontrar otros (España y la bebida por ejemplo), creemos se rigen por la misma actitud.

Ahora, en este primer caso, se aprecia según Bajtín que el autor no puede encontrar un punto de apoyo válido y estable fuera del personaje. Los momentos conclusivos por lo mismo los ubica fuera del personaje y tienen un carácter casual, poco fundamentado e inseguro. Para quien haya leído la novela se hace evidente que precisamente existe una ambigüedad en medio del lenguaje directo y objetivo de Hemingway: no se sabe bien qué pa. sa y quién centra la acción. Pareciera ser al comienzo Cohn, pero luego la aparición de Barnes y el tono induce a suponerlo el protagonista, sin embargo existe Ashley, que tampoco tiene un ingreso narrativo definido pues aparece entre un grupo de individuos colaterciles, cuya importancial no podemos soslayar.

Fiesta tiene precisamenet esa funcionalidad conclusiva no en Bames o Ashley sino en los personajes secundarios cuya participación se torna en el desarrollo de la cección casual, poco fundamentada (hay muchas interroganles que quedan sueltas) e insegura (17). Esta inseguridad es explícita y, sobre todo, recogida por la críica al inientar establecer entre ung gmplia variedad de apreciaciones el protagonismo central de la Bbra.

Así Baker sostúde gqueujake Bdinesives' el "protagonista de la obra, describiéndolo como "un aporreado, no vencido" (18) y Young literalmente afirma "The sun also rises nos vuelve a presentar al HEROE que aquí se llama Jake Barnes" (19). Hemingway, por su lado, se refirió a la novela como "una condenada tragedia con el terruño como héroe eterno" (20), opinión que comparte Wirt Williams (21). Otros resallan el papel de Bret Ashley (22) o Roberl Cohn (23).

(17) Young comenta lo siguiente de Fiesta: "Nada en el libro lleva a ninguna parte". Ob. Cit. p. 15.

(18) BAKER, Carlos: E. Hemingway: El escritor como artista.

(19) Ob. cit., p. 14,

(20) E.H. a Maxwell Perkins, Nov. 19, 1926. Cit. por Baker.

(21) El axíe trágico de E.H.

(22) Mark Spilka: "La muerte del amor". En: Hemingway y sus criticos New York, Hill y Wang, 1961.

(23) Harold Loeb "Cómo era" N.Y.. Criterion, 1959. 
Cualquiera que sea el protagonista 0 , como nosotros suponemos, siendo Jake Barnes, lo cierto es que este héroe se impone a E.H., se apropia de él y se mueve con una postura incierta y prestigiosa, tal vez porque es él mismo, su propia proyedición discursiva.

La segunda actitud la define Bajtín como aquella donde el autor se apropia del personaje, introduciendo momentos conclusivos. El personaje al ser autobiográlico asume al autor en forma conclusiva, adoptando el discurso y su alma para luego superarla, rebasando por inadecuada la totalidad. Es un personaje inconcluso.

Esto se manifiesta en cierto misterio interior que no puede ser expresado y que el personaje contrapone a la totalidad concluida del autor.

Frederic Henry, el protagonista de Adios a las armas entra perfectamente dentro de esta definición. Luego de un Jake Barnes demasiado parco, que casi no habla de sí mismo y no posee monó. logos interiores, sucede un tipo Eomo Henry que expresa su visión en múltiples pasajes, pero sin embargo se constituye en un personaje lleno de aspeatos extraños y misteriosos: su ligazón con la guerra siendo extranjero, su no creer en el amor, su religiosidad. Y un largo etcéterc.

Lo que expresa Bajtín se aplica casi exactamente a Federico Henry y lo expresa cabalmenle: "¿Ustedes creen que yo totalmente estoy aquí -parece que dijera el personaje-, que están viendo mi totalidad? Ustedes no pueden ver, ni oír, ni saber aquello que es lo más importante para mí" (24).

La crítica norteamericana ha señalado, al respecto, la re:a-

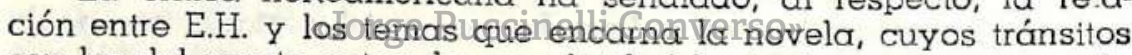
son los del propio autor: la complicidad hasta la amargura con la guerra, del prostíbulo hasta el verdadero amor, la mezcla de pesimismo y el ideal, la sensación de tragedia y la desesperación (25).

Por último, Bajtín, señala la actitud final: "el personaje es su propio autor, comprende su propia vida istéticamente, está representando cierto papel" y "es cutosuficiente y concluido de una manera total" (26).

En esta opción se encuadra Santiago, el anciano pescador de El viejo y el mar. Sus momentos conclusivos están fuera de la conciencia del peisonaje y son utilizados para heroizar. El plano de fondo, lo invisible y lo desconocido que transcurre a espaldas del personaje le permiten destacar y le otorgan su carácter de héroe.

Esa relación causal con el medio lleva a Santiago precisamente a enfrentar su realidad como si tuviera que cumplir una obliges-

(24) BAJTIN, M.M.: Ob. cit., p, 27.

(25) cf. P. YOUNG.

(26) BAJTIN, M.M. Ob. cit., p. 27. 
ción consigo mismo, en el buen sentido de la palabra éste es el personaje d Hemingway que mejor cumple su papel de héroe.

Hemingway y el viejo mar de las palabras.

De lo expuesto hasta aquí podemos deducir, no sólo un nuevo planteamiento para abordar la narrativa de Hemingway, sino es. pecialmente que el proceso de ampliación estilística del autor que lleva a un lento distanciamiento con el personaje, adquiere su concreción en El viejo y el mar.

Cabe, sin embargo, preguntarnos el por qué de la actitud de la crítica norteamericana de relacionar en un mismo plano lo biográfico y lo literario.

Creemos que ello se explica por la continuidad de los viejos postulados de New Criticism, que busca hacer de la crítica un ccto de valoración de la realidad, eludiendo aspectos eruditos o formales. Todo ello es evidente sobre todo cuando en la valoración de Hemingway se habla de la individualidad y poco, o casi nada, de su inserción en la tradición, dejando su aporte o ruptura al nivel del acierto individualista. La crítica se convierte así en la apología del egocentrismo y su método la fácil paráfrasis de lo evidente o supuesto.

La ceguera de la crítica norteamericana en tomo a $E I$ viejo $y$ el mar, obra que precisamente logra una conclusión mayor, se explica así también por el hecho de la incapacidad de los enfoques de precisar el sentido de aquella obra alejada de la biografía del autor.

La dimensión Hifraria de Emest Hemingwa no debe establecerse en base a la influencia dèinsul vidanpersonal y su imagen. Es innegable que la reducción de su obra a ciertos personajes y motivos, que une niveles de cuento y novela, tiene su correlato en la desatención de los aspectos textuales del efecto estético. Punto en el cual la inadecuada correlación entre aulor y personaje, dan a la obra conjunta de E.H. cierto matiz imperfecto, estructuralmente inconclusa. Ello no debe llevarnos a negarle los méritos que sin lugar a dudas tiene, no sólo dentro de la literatura norteamericana sino mundial, pero creo también sirve para mirar con otros ojos al cuentista, cuya perspectiva y dimensión tal vez el bullicioso élan del novelista ha opacado.

Aunque pasen los años, la vieja barcaza del viejo "Papa" sur cará el inmenso mar de las palabras intentando diferenciarse de su propia voz, pues "un acontecer estético puede darse únicamente cuando hay dos participantes, presupone la existencia de dos conciencias que no coinciden" (27) en cuya lucha y autonomía se establece la grandeza o la heroicidad del propio hombre.

(27) Ibid., p. 28. 